2016 were included. Patients were divided into CTEPH/CTED groups and their pathways followed until July 2017.

Results Of the 316 referrals to the PEA MDT there were 246 cases of CTEPH, 46 of CTED and 24 with alternate diagnoses. Age range of referrals was 22-88 years (mean 59 years). $51 \%$ were male. The majority of CTEPH cases had technically operable disease $(n=200,81 \%)$ with 130 proceeding to PEA (65\%). 53\% of all CTEPH cases were operated on (figure 1). Median time from first MDT discussion to PEA was 230 days with a median surgical waiting list time of 115 days. 11 patients required ongoing targeted therapy post-operatively. Of the 70 individuals with an operable disease distribution who did not proceed to PEA, 34 patients chose to decline surgery and 36 were rejected by the surgical team due to co-morbid conditions $(n=28)$ or limited symptoms $(n=8)$. Two patients with distal CTEPH underwent Balloon Pulmonary Angioplasty (BPA). Remaining patients were medically managed with targeted therapy. Of CTED cases, 28 (61\%) had an operable disease distribution with 8 (29\%) proceeding to PEA. $17 \%$ of total CTED referrals were operated on.

Conclusion This patient pathway mapping is the first undertaken for any national CTEPH/CTED cohort. The majority of UK CTEPH cases suitable for PEA are operated on (65\%). The duration of time between MDT referral and PEA is reflective of disease complexity and decision-making process. We observe a group of highly selected individuals with Chronic Thromboembolic Disease (CTED) without pulmonary hypertension who undergo PEA after careful consideration of their symptoms and operative risk.

\section{S52 COMPUTED TOMOGRAPHY IN THE DIAGNOSIS OF LEFT HEART DISEASE IN PATIENTS WITH SUSPECTED PULMONARY HYPERTENSION}

${ }^{1} \mathrm{~B}$ Currie, ${ }^{1} \mathrm{C}$ Johns, ${ }^{1} \mathrm{M}$ Chin, ${ }^{2} \mathrm{CA}$ Elliot, ${ }^{2} \mathrm{RA}$ Condliffe, ${ }^{2} \mathrm{~A}$ Charalampopolous, ${ }^{1} \mathrm{~S}$ Rajaram, ${ }^{1} \mathrm{JM}$ Wild, ${ }^{1} \mathrm{DG}$ Kiely, ${ }^{1} \mathrm{AJ}$ Switt. ${ }^{1}$ University of Sheffield, Sheffield, UK; ${ }^{2}$ Sheffield Pulmonary Vascular Disease Unit, Sheffield, UK

\subsection{6/thoraxjnl-2017-210983.58}

Background Identification of patients with left heart disease (LHD) as the cause of pulmonary hypertension is challenging, developing a tool that can identify these patients would reduce unnecessary referral for investigation at specialist centres and may reduce the burden of invasive investigations. The aim was to investigate the capability of computed tomography (CT)-derived metrics for the diagnosis LHD in a cohort of patients with suspected pulmonary hypertension.

Methods Patients with suspected pulmonary hypertension who underwent CT and RHC were identified. Derivation and validation cohorts were randomly constructed to derive and test a binary logistic regression model. All image analysis took place on PACS system blinded to patient's cardiac catheter data and diagnosis. CT measurements of the cardiac chambers and vessels were taken. LHD was defined by increased pulmonary arterial wedge pressure (PAWP) $\geq 15 \mathrm{mmHg}$. A second threshold of $18 \mathrm{mmHg}$ defined more advanced LHD. Backward binary logistic regression in a derivation cohort identified a model for predicting Group $2 \mathrm{PH}$. This model was tested in the validation cohort and compared to individual CT derived variables using receiver operating characteristic curve analysis and chi-square.

Results The CT scans were from 66 different centres and 446 patients were identified, derivation cohort $(n=235)$ and validation cohort $(n=211)$. Left atrial area was found to be most significant individual predictor of elevated PAWP, area under curve (AUC) 0.86, p $<0.001$, the accuracy was higher for identification of PAWP $\geq 18$, AUC 0.87, $\mathrm{p}=0.87$, $\mathrm{p}<0.001$. Derived regression models did not add diagnostic value AUC in validation cohort 0.87, $\mathrm{p}<0.001$. A limit for enlarged left atrial area was set at $27.5 \mathrm{~cm}^{2}$. This had sensitivity $65 \%$ and specificity $90 \%$ in predicting Group $2 \mathrm{PH}$ using PAWP $\geq 18 \mathrm{mmHg}$ as a threshold.

Conclusions CT derived left atrial area is a specific predictor of LHD in suspected pulmonary hypertension. Composite models did not increase diagnostic value. Left atrial area on CT may be a useful tool for diagnosing PH-LHD and may reduce unnecessary referrals to specialist $\mathrm{PH}$ centres and reduce the number of invasive investigations.

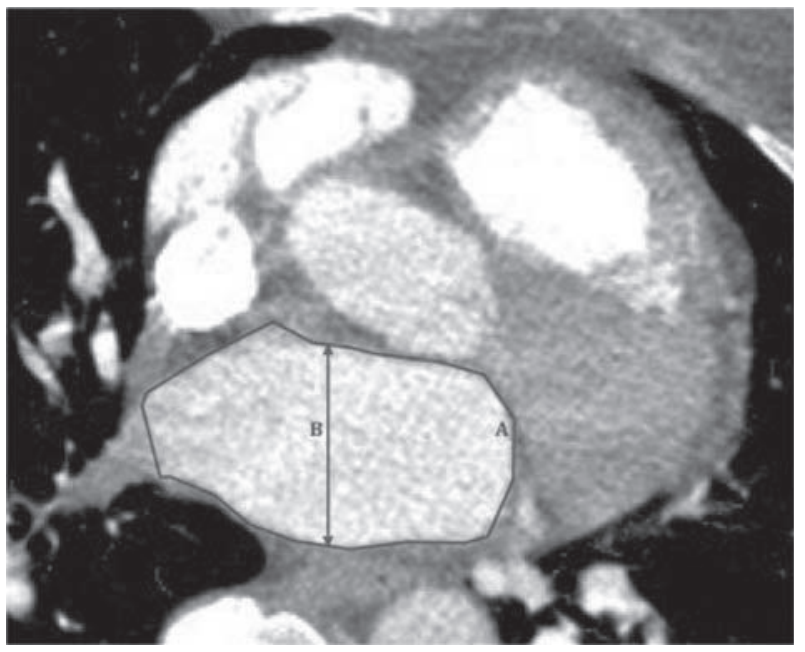

Abstract S52 Figure 1

\section{From diagnosis to treatment in interstitial lung disease}

\section{S53 \\ MARKED SMALL AIRWAY DYSFUNCTION AND CONSEQUENT AIR-TRAPPING CHARACTERISE CHRONIC HYPERSENSITIVITY PNEUMONITIS (CHP) BUT NOT IDIOPATHIC PULMONARY FIBROSIS (IPF)}

${ }^{1} \mathrm{M}$ Bonini, ${ }^{1} \mathrm{O}$ Usmani, ${ }^{2} \mathrm{~A}$ Pacini, ${ }^{2} \mathrm{I}$ Menichini, ${ }^{1} \mathrm{~S}$ Ward, ${ }^{3} \mathrm{~S}$ Walsh, ${ }^{1} \mathrm{C}$ Daccord, ${ }^{1} \mathrm{C}$ Minelli, ${ }^{2} \mathrm{M}$ Brunori, ${ }^{1} \mathrm{AU}$ Wells, ${ }^{1} \mathrm{~F}$ Chua. 'Royal Brompton Hospital, London, UK; ${ }^{2}$ 'Sapienza' University of Rome, Rome, Italy; ${ }^{3}$ Kings College Hospital, London, UK

\subsection{6/thoraxjnl-2017-210983.59}

Introduction CHP, the major differential diagnosis for IPF, has varied radiological features that can confound CT agreement in up to a third of cases of fibrotic interstitial lung disease presenting to multidisciplinary panels. Given that the pathological basis of CHP incorporates diffuse bronchiolitic abnormalities, we hypothesised that differences in small airway dysfunction and resultant air-trapping might help refine a multi-domain approach to distinguishing CHP from IPF.

Methods Analysis of lung function records of individuals with multidisciplinary diagnoses of CHP and IPF ( $n=118$ in each group) with matched disease severity (\% predicted FVC: 69.3 \pm 21.9 vs. $73.8 \pm 16.2$ respectively, $\mathrm{p}=\mathrm{n} / \mathrm{s}$ and $\%$ predicted 
TLco: $39.2 \pm 14.8$ vs $39.9 \pm 13.1$ respectively, $p=n / s$ ) at the Royal Brompton Hospital was undertaken. Indices relating to airflow, dynamic and static lung volumes were compared. Data were analysed using parametric tests following testing for normal distribution and expressed as mean \pm SD. A pvalue $<0.05$ was considered statistically significant.

Results Compared to patients with IPF, the CHP cohort was characterised by considerably worse small airway impairment as denoted by significantly lower\%-predicted values of midexpiratory flow $\left(\mathrm{MEF}_{25 / 50 / 75} ; \mathrm{p}<0.0001\right)$, forced expiratory volume at $3 \mathrm{~s}\left(\mathrm{FEV}_{3} ; \mathrm{p}<0.05\right)$, volumic inspiratory and expiratory airway conductance $\left(\mathrm{G}_{\mathrm{AW}}\right)$ as well as resistance $\left(\mathrm{R}_{\mathrm{AW}}\right)$ $(\mathrm{p}<0.0001)$. In contrast, indicators of greater air-trapping including residual volume/RV and the RV/TLC ratio were increased in CHP ( $p<0.0001$ and $p<0.0001$ respectively) while contraction of total lung capacity occurred to a lesser extent in this group $(p=0.01)$. The gas transfer coefficient $/$ Kco was lower in CHP than in IPF $(p=0.01)$.

Conclusion Although both diseases result in restrictive pulmonary physiology, patients with CHP have demonstrably worse small airway function than those with IPF, as evaluated by a range of flow and volume determinants. Consequent upon these changes, a higher degree of air-trapping is evident in the CHP cohort. The radiologic correlates of such observations, namely bronchocentric fibrosis and lobular air-trapping, are widely recognised in CHP. In future, integration of a more disease-specific physiologic profile with detailed assessment of disease behaviour, volumetric radiology and dynamic small airway tests may enhance the accuracy of diagnosing $\mathrm{CHP}$.

\section{S54 AUTOMATING THE ANALYSIS OF THORACIC CT SCANS IN CYSTIC LUNG DISEASE}

${ }^{1} V$ Maharajan, ${ }^{2} \mathrm{~S}$ Karia, ${ }^{1} E R$ Maher, ${ }^{1}$ SN Taraskin, ${ }^{3}$ SR Johnson, 'SJ Marciniak. 'University of Cambridge, Cambridge, UK; ${ }^{2}$ Addenbrooke's Hospital, Cambridge, UK; ${ }^{3}$ University of Nottingham, Nottingham, UK

\subsection{6/thoraxjn--2017-210983.60}

Introduction Certain disorders of the lung, such as Birt-HoggDubé syndrome (BHD) and lymphangioleiomyomatosis (LAM), are characterised by the presence of multiple pulmonary cysts. Radiological analysis using thoracic computed tomography (CT) is the mainstay of diagnosis and follow-up of these disorders. The rate of change of the cysts contributes to therapeutic decisions including the prescription of potentially toxic therapies, most notably mTOR inhibitors in LAM. At present, cyst parameters including their location, size, shape and number are determined by the review of CT images by radiologists. Despite expert training, this process is prone to human error and susceptible to inter-observer disparity.

Objective We wished to determine if automation of cyst analysis could provide robust data to aid the radiologists in their reporting of thoracic CT scans.

Methods and Results Software was developed using $\mathrm{C}++$ to extract data from standard Digital Imaging and Communications in Medicine (DICOM) CT files. For each scan, voxels in lung parenchyma or cysts were detected by radiodensity being in the range from $-935 \mathrm{HU}$ to $-610 \mathrm{HU}$ or below -935 $\mathrm{HU}$, respectively. The $3 \mathrm{D}$-cyst boundaries were identified by means of novel recursive algorithm (figure 1). Trachea and airways were automatically detected and excluded from further analysis. Number of cysts per patient was recorded and each cyst analysed in terms of volume, spatial location, sphericity and cylindricity (calculated by using eigenvalues of gyration tensor for corresponding cyst) and opacity. The software was calibrated empirically through iterative adjustment of the above threshold values and comparison with scores generated by an expert thoracic radiologist thus enabling the reliable differentiation of cysts from noise. As proof-of-principle, the scans of 10 individuals with BHD and 10 with LAM were analysed in a blinded manner by the computer and compared with independent radiology reports.

Conclusion Automated image analysis provides a new set of objective cyst parameters and offers added value to the thoracic radiology reporting process. Future studies will determine the relative sensitivities of human vs. automated CT analysis in the diagnosis and monitoring of cystic lung diseases including BHD and LAM.

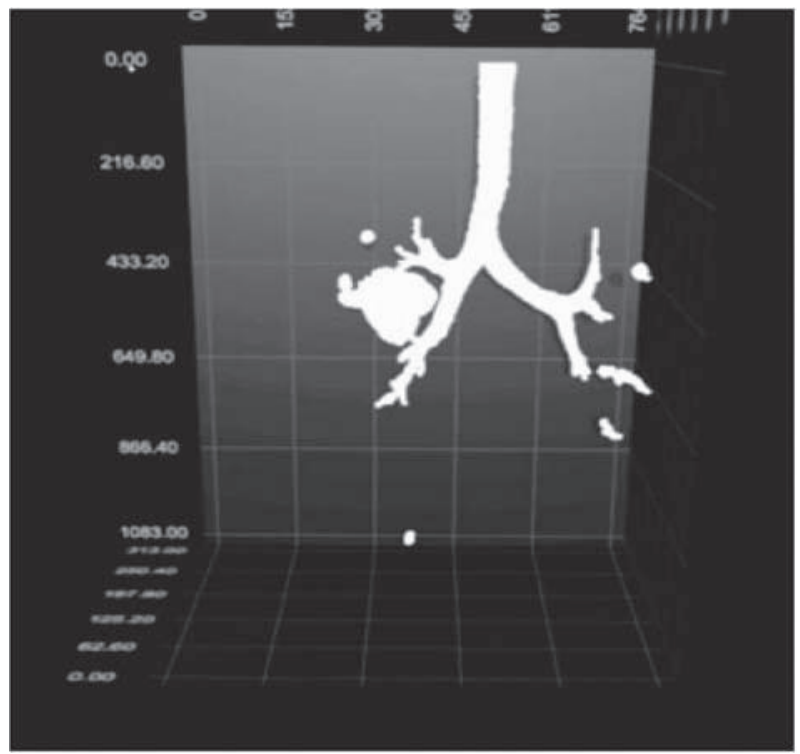

Abstract S54 Figure 1 Automated detection of lung cysts. 3D rendering of the thoracic $\mathrm{CT}$ scan of an individual with BHD following automated detection of cysts but prior to deletion of airways. Note irregular cysts with a preponderance below level of the carina.

\section{S55 DERIVATION AND VALIDATION OF A SIMPLE LONGITUDINAL SCORE WHICH STRONGLY PREDICTS MORTALITY IN INTERSTITIAL LUNG DISEASE (ILD) ASSOCIATED PULMONARY HYPERTENSION (ILD-PH)}

${ }^{1} \mathrm{SRB}$ Bax, ${ }^{1} \mathrm{C}$ Breedy, ${ }^{1} \mathrm{~K}$ Dimopoulos, ${ }^{1} \mathrm{~A}$ Kempny, ${ }^{1} \mathrm{~A}$ Devaraj, ${ }^{2} \mathrm{~S}$ Walsh, ${ }^{1} \mathrm{~J}$ Joseph, ${ }^{3} \mathrm{~A}$ Nair, ${ }^{4} \mathrm{G}$ Kier, ${ }^{1} \mathrm{M}$ Kokosi, ${ }^{1} \mathrm{C}$ Harries, ${ }^{1} \mathrm{~V}$ Kouranos, ${ }^{1} \mathrm{C} \mathrm{McC}$ abe, ${ }^{1} \mathrm{~W} \mathrm{Li},{ }^{5} \mathrm{M}$ Wilde, ${ }^{1} \mathrm{AU}$ Wells, ${ }^{1}$ LC Price*, ${ }^{1} \mathrm{SJ}$ Wort*. ${ }^{1}$ Royal Brompton Hospital, London, UK; ${ }^{2}$ Kings College Hospital Foundation Trust, London, UK; ${ }^{3}$ Guy's and St Thomas' NHS Foundation Trust, London, UKi ${ }^{4}$ Princess Alexandra Hospital, Brisbane, Australia; ${ }^{5}$ Surrey and Sussex NHS Trust, London, UK; *Joint final author

\subsection{6/thoraxjnl-2017-210983.61}

Introduction Pulmonary hypertension commonly occurs in ILD, and is a malignant prognostic factor. Predicting mortality in this group remains problematic. We hypothesised that a combination of baseline demographics and longitudinal change in PFT's and the biomarker brain-natriuretic peptide (BNP) would predict mortality in ILD-PH.

Methods Demographics, ILD subtype, PFTs, echocardiogram, and CTs were reviewed in consecutive patients undergoing 